CONFORMAL GEOMETRY AND DYNAMICS

An Electronic Journal of the American Mathematical Society

Volume 6, Pages 61-73 (August 7, 2002)

S $1088-4173(02) 00085-1$

\title{
TRANSVERSELY PROJECTIVE STRUCTURES ON A TRANSVERSELY HOLOMORPHIC FOLIATION, II
}

\author{
INDRANIL BISWAS
}

\begin{abstract}
Given a transversely projective foliation $\mathcal{F}$ on a $C^{\infty}$ manifold $M$ and a nonnegative integer $k$, a transversal differential operator $\mathcal{D}_{\mathcal{F}}(2 k+1)$ of order $2 k+1$ from $N^{\otimes k}$ to $N^{\otimes(-k-1)}$ is constructed, where $N$ denotes the normal bundle for the foliation. There is a natural homomorphism from the space of all infinitesimal deformations of the transversely projective foliation $\mathcal{F}$ to the first cohomology of the locally constant sheaf over $M$ defined by the kernel of the operator $\mathcal{D}_{\mathcal{F}}(3)$. On the other hand, from this first cohomology there is a homomorphism to the first cohomology of the sheaf of holomorphic sections of $N$. The composition of these two homomorphisms coincide with the infinitesimal version of the forgetful map that sends a transversely projective foliation to the underlying transversely holomorphic foliation.
\end{abstract}

\section{INTRODUCTION}

Let $M$ be a $C^{\infty}$ manifold equipped with a $C^{\infty}$ foliation $\mathcal{F}$ of codimension two. So we can cover $M$ by a collection of open subsets $U_{j}, j \in J$, such that for each $j$ there is a $C^{\infty}$ submersion $\phi_{j}: U_{j} \longrightarrow \mathbb{C}$ with the property that the leaves of the foliation $\left.\mathcal{F}\right|_{U_{j}}$ on $U_{j}$ coincide with the fibers of the map $\phi_{j}$. Let $V$ be a $C^{\infty}$ vector bundle over $M$ equipped with a flat partial connection along $\mathcal{F}$. This means that for each $j \in J$ there is a $C^{\infty}$ vector bundle $V_{j}$ over $\phi_{j}\left(U_{j}\right)$ and an isomorphism $\psi_{j}:\left.V\right|_{U_{j}} \longrightarrow \phi_{j}^{*} V_{j}$ such that $\psi_{j^{\prime}} \circ \psi_{j}^{-1}$ over $U_{j} \cap U_{j^{\prime}}$ is the pullback of an isomorphism $\tau_{j, j^{\prime}}$ of $\left.V_{j}\right|_{\phi_{j}\left(U_{j} \cap U_{j^{\prime}}\right)}$ with $\left.V_{j^{\prime}}\right|_{\phi_{j^{\prime}}\left(U_{j} \cap U_{j^{\prime}}\right)}$ over the (unique) map $\sigma_{j, j^{\prime}}$ from $\phi_{j}\left(U_{j} \cap U_{j^{\prime}}\right)$ to $\phi_{j^{\prime}}\left(U_{j} \cap U_{j^{\prime}}\right)$ that satisfies the identity $\sigma_{j, j^{\prime}} \circ \phi_{j}=\phi_{j^{\prime}}$.

The foliation $\mathcal{F}$ is said to have a transversely holomorphic structure (respectively, transversely projective structure) if the covering can so chosen that $\phi_{j^{\prime}} \circ \phi_{j}^{-1}$ is a holomorphic (respectively, fractional linear) map for every pair $j, j^{\prime} \in J$. (See [8] for projective structures on a Riemann surface.)

Let the foliation $\mathcal{F}$ be equipped with a transversely holomorphic structure. The above vector bundle $V$ is called transversely holomorphic if each $V_{j}$ has a holomorphic structure and each isomorphism $\tau_{j, j^{\prime}}$ is a holomorphic structure preserving (which makes sense since each $\phi_{j^{\prime}} \circ \phi_{j}^{-1}$ is a holomorphic map). For example, the normal bundle $N=T M / \mathcal{F}$ is a transversely holomorphic line bundle.

In [1, we described the space of all transversely projective structures on a given transversely holomorphic foliation. In this continuation of 1], we consider the infinitesimal deformations of a transversely projective structure without fixing the underlying transversely holomorphic structure and the homomorphism from this

Received by the editors October 22, 2001 and, in revised form, June 24, 2002.

2000 Mathematics Subject Classification. Primary 37F75; Secondary 53B10.

(C)2002 American Mathematical Society 
infinitesimal deformation space to the infinitesimal deformations of the underlying transversely holomorphic structure defined by the forgetful map that sends a transversely projective foliation to the underlying transversely holomorphic foliation.

In Section [3, we construct transversal jet bundles $J_{\mathcal{F}}^{k}(V)$ of $V$ and transversal differential operators $\operatorname{Diff}_{\mathcal{F}}^{k}(V, W)$, where $W$ is another transversely holomorphic vector bundle. If $\mathcal{F}$ is equipped with a transversely projective structure, then for each $k \geq 0$ we construct a transversal differential operator

$$
\mathcal{D}_{\mathcal{F}}(2 k+1) \in \Gamma\left(M ; \operatorname{Diff}_{\mathcal{F}}^{2 k+1}\left(N^{\otimes k}, N^{\otimes(-k-1)}\right)\right)
$$

over $M$ of order $2 k+1$. This operator depends on the transversely projective structure.

Let $\mathcal{W}(\mathcal{F})$ denote the sheaf on $M$ defined by the kernel of the operator $\mathcal{D}_{\mathcal{F}}(3)$. It turns out that $\mathcal{W}(\mathcal{F})$ is the local system on $M$ associated to a flat connection on the vector bundle $J_{\mathcal{F}}^{2}(N)$ (Lemma 5.1). We construct a homomorphism from the space of all infinitesimal deformations of the transversely projective structure to $H^{1}(M, \mathcal{W}(\mathcal{F}))$ (Theorem 5.3). We will denote this homomorphism by $\zeta$.

Since the domain of the operator $\mathcal{D}_{\mathcal{F}}(3)$ is $N$, we have a natural inclusion of $\mathcal{W}(\mathcal{F})$ in the sheaf, denoted by $\mathcal{C}(1)$, of (locally defined) holomorphic sections of $N$. Therefore, we have a homomorphism

$$
\delta: H^{1}(M, \mathcal{W}(\mathcal{F})) \longrightarrow H^{1}(M, \mathcal{C}(1))
$$

(see Proposition 5.2). The space of all infinitesimal deformations of the transversely holomorphic foliation has a natural homomorphism to $H^{1}(M, \mathcal{C}(1))$. This homomorphism will be denoted by $\zeta^{\prime}$.

The forgetful map that sends a transversely projective foliation to the underlying transversely holomorphic foliation defines a homomorphism from the infinitesimal deformations of a transversely projective foliation to the infinitesimal deformations of the underlying transversely holomorphic foliation. The composition of this homomorphism with $\zeta^{\prime}$ coincides with $\delta \circ \zeta$ (Theorem 5.3.

\section{Preliminaries}

Let $M$ be a $C^{\infty}$ manifold of dimension $d+2$, with $d \geq 0$. Let

$$
\mathcal{F} \subset T M
$$

be a $C^{\infty}$ vector subbundle of rank $d$ defining a foliation. In other words, $\mathcal{F}$ is closed under the Lie bracket operation on the locally defined smooth vector fields. Let

$$
N:=\frac{T M}{\mathcal{F}}
$$

be the normal bundle. The inclusion map of $\mathcal{F}$ in $T M$ will be denoted by $i$ and the projection of $T M$ to $N$ will be denoted by $q$.

The Lie bracket operation on the (local) sections of $T M$ induces a partial connection on $N$ known as the Bott partial connection. We will first recall the definition of a general partial connection.

Let $V$ be a $C^{\infty}$ vector bundle over $M$. A partial connection on $V$ is a first order differential operator

$$
D: V \longrightarrow \mathcal{F}^{*} \otimes V
$$


satisfying the Leibniz identity which says that for every (locally defined) smooth section $s$ of $V$ and every (locally defined) $C^{\infty}$ function $f$ on $M$, the equality

$$
D(f s)=f D(s)+i^{*} d f \otimes s
$$

holds, where $i^{*}$ is the dual of the inclusion map $i$ of $\mathcal{F}$.

If $D^{\prime}$ is a partial connection on $V^{\prime}$, then $D \otimes \operatorname{Id}_{V^{\prime}}+\mathrm{Id}_{V} \otimes D^{\prime}$ is a partial connection on $V \otimes V^{\prime}$. Similarly, $D$ induces a partial connection on the dual vector bundle $V^{*}$. A section $s$ of $V$ is called flat if $D(s)=0$.

The curvature $D \circ D$ of the partial connection $D$ is a $C^{\infty}$ section of $\bigwedge^{2} \mathcal{F}^{*} \otimes$ $\operatorname{End}(V)$. If the curvature is identically zero, then $D$ will be called flat.

To describe the Bott partial connection, consider

$$
D_{B}: N \longrightarrow \mathcal{F}^{*} \otimes N
$$

defined by $\left\langle D_{B}(s), t\right\rangle=q([t, s])$, where $\langle-,-\rangle$ is the contraction of $\mathcal{F}^{*}$ and $\mathcal{F}$ (so both sides are sections of $N$ ). It is easy to see that $D_{B}$ is a partial connection on $N$. The Jacobi identity for Lie bracket implies that the Bott connection $D_{B}$ is flat. The partial connection on any tensor power of $N$ induced by $D_{B}$ will also be called the Bott partial connection.

A transversely holomorphic structure on the foliation $\mathcal{F}$ is a $C^{\infty}$ section $J$ of the vector bundle $\operatorname{End}(N)$ satisfying the following conditions:

(1) $J^{2}=-\operatorname{Id}_{N}$;

(2) $J$ is flat with respect to the partial connection on $\operatorname{End}(N)$ induced by the Bott partial connection.

Since an almost complex structure on a two dimensional manifold is automatically integrable, the above definition of a transversely holomorphic structure coincides with its usual definition (see [9, [4], [5, 6], 7]). We recall that a transversely holomorphic structure is alternatively defined by a data $\left\{U_{j}, \phi_{j}\right\}_{j \in J}$, where $\left\{U_{j}\right\}_{j \in J}$ is an open covering of $M$ and $\phi_{j}: U_{j} \longrightarrow \mathbb{C}$ are submersions such that:

(1) for each $j \in J$, the kernel of the differential $d \phi_{j}$ coincides with $\left.\mathcal{F}\right|_{U_{j}}$ and

(2) for each pair $j, j^{\prime}$, there are holomorphic maps

$$
g_{j, j^{\prime}}: \phi_{j}\left(U_{j} \cap U_{j^{\prime}}\right) \longrightarrow \phi_{j^{\prime}}\left(U_{j} \cap U_{j^{\prime}}\right)
$$

satisfying the equality $g_{j, j^{\prime}} \circ \phi_{j}=\phi_{j^{\prime}}$ of maps on $U_{j} \cap U_{j^{\prime}}$.

Since the kernel of $d \phi_{j}$ coincides with $\left.\mathcal{F}\right|_{U_{j}}$, the normal bundle $\left.N\right|_{U_{j}}$ gets identified with $\phi_{j}^{*} T \mathbb{C}$, the tangent bundle of the complex plane. Since each $g_{j, j^{\prime}}$ is a holomorphic map, the almost complex structure $J_{j}$ on $\left.N\right|_{U_{j}}$ induced by the holomorphic structure of $T \mathbb{C}$ using this identification is independent of $j$. In other words, $J_{j}$ and $J_{j^{\prime}}$ coincide over $U_{j} \cap U_{j^{\prime}}$. Therefore, we get a transversely holomorphic structure in the previous sense. Conversely, if we take a local slice $f: U \longrightarrow M$ transversal to $\mathcal{F}$, where $U$ is an open subset of $\mathbb{R}^{2}$, then using the obvious identification of $T U$ with $f^{*} N$, an almost complex structure $J$ on $N$ induces a complex structure on $U$.

A transversely projective structure is a transversely holomorphic structure satisfying the further condition that the above maps $g_{j, j^{\prime}}$ are all Möbius transformations [1]. We recall that Möbius transformations are automorphisms of $\mathbb{C P}^{1}$; that is, functions of the form $z \longmapsto(a z+b) /(c z+d)$, with $a d-b c=1$. Therefore, a transversely projective structure has an underlying transversely holomorphic structure. If the condition that $g_{j, j^{\prime}}$ are all Möbius transformations is satisfied, then such a locally defined map $\phi_{j}$ will be called compatible with the transversely projective structure defined by them. 
Examples of transversely projective structures can be found in [3] and [10. Particularly in [10, p. 190, Example 1.1] and [3, p. 630, Theorem C] interesting classes of transversely projective structures are constructed.

Take a transversely holomorphic structure $J$ on $\mathcal{F}$. The automorphism $J$ makes $N$ a $C^{\infty}$ complex line bundle.

For $k<0$, by $N^{\otimes k}$ we will mean the line bundle $\left(N^{*}\right)^{\otimes-k}$ over $M$. We recall that the Bott partial connection on $N$ induces a flat partial connection on $N^{\otimes k}$ for each $k \in \mathbb{Z}$. Consider the sheaf of sections of the complex line bundle $N^{\otimes k}, k \in \mathbb{Z}$, flat with respect to the Bott partial connection. Denote this sheaf by $\mathcal{S}(k)$.

Let $\overline{N^{*}}$ denote the complex line bundle with complex structure defined by the conjugation of the complex structure of $N^{*}$. The transversely holomorphic structure induces a natural analog of the Dolbeault operator

$$
\bar{\partial}_{k}: \mathcal{S}(k) \longrightarrow \overline{N^{*}} \otimes \mathcal{S}(k)
$$

that satisfies the Leibniz identity which says that

$$
\bar{\partial}_{k}(f s)=f \bar{\partial}_{k}(s)+\bar{\partial} f \otimes s,
$$

where $f$ is a locally defined smooth function constant along the leaves (i.e., $\langle d f, \mathcal{F}\rangle=$ 0 ) and $s$ is a section $\mathcal{S}(k)$. Note that the condition $\langle d f, \mathcal{F}\rangle=0$ ensures that $f s$ is also a section of $\mathcal{S}(k)$ and $\bar{\partial} f$ gives a section of $\overline{N^{*}}$. The Dolbeault operator $\bar{\partial}$ coincides with $\bar{\partial}_{0}$ for the obvious identification of $\mathcal{S}(0)$ with the sheaf of locally defined smooth functions on $M$ that are constant along the leaves.

Let $\mathcal{C}(k) \subset \mathcal{S}(k)$ be the subsheaf defined by the kernel of $\bar{\partial}_{k}$. We will denote by $\mathcal{V}(k)$ the complex vector space defined by the space of all global sections of $\mathcal{C}(k)$. In other words, $s \in \mathcal{V}(k)$ if $s$ is a section of $\mathcal{S}(k)$ over $M$ with $\bar{\partial}_{k} s=0$.

Let $J$ be a transversely holomorphic structure on $\mathcal{F}$ admitting a transversely projective structure (the underlying transversely holomorphic structure coincides with $J$ ). It may be noted that a general transversely holomorphic structure need not admit a transversely projective structure. The space of all transversely projective structures on $\mathcal{F}$ that has the fixed underlying transversely holomorphic structure $J$ is an affine space for $\mathcal{V}(-2)$ [1, Theorem 2.6]. It is known that $\mathcal{V}(-2)$ is finite dimensional if $M$ is compact [6], 4].

Let $V$ be a $C^{\infty}$ complex vector bundle of rank $n$ over $M$ equipped with flat partial connection $D(V)$ with respect to $\mathcal{F}$. Let $\mathcal{S}(V)$ denote the sheaf of sections of $V$ flat with respect to $D(V)$. A transversely holomorphic structure on $V$ (with respect to $J$ ) is a differential operator

$$
\bar{\partial}_{V}: \mathcal{S}(V) \longrightarrow \overline{N^{*}} \otimes \mathcal{S}(V)
$$

satisfying the Leibniz identity $\bar{\partial}_{V}(f s)=f \bar{\partial}_{V}(s)+\bar{\partial} f \otimes s$, where $f$ is a locally defined smooth function constant along the leaves and $s$ is a section $\mathcal{S}(V)$. Since we are in complex dimension one, no extra integrability condition on $\bar{\partial}_{V}$ is required since it is automatically integrable.

A (locally defined) section $s$ of $V$ is called holomorphic if it is a section of $\mathcal{S}(V)$ (that is, constant along the leaves) and $\bar{\partial}_{V}(s)=0$. In particular, if we set $V$ to be $N^{\otimes k}$, then a holomorphic section is a (local) section of the sheaf $\mathcal{C}(k)$ defined earlier.

It is easy to see that a transversely holomorphic structure on $V$ can alternatively be defined as follows. There is an open covering $\left\{U_{i}\right\}_{i \in I}$ of $M$ satisfying the conditions given the definition of a transversely holomorphic structure, and on each $U_{i}$ 
there is a $C^{\infty}$ isomorphism

$$
\psi_{i}:\left.V\right|_{U_{i}} \longrightarrow \mathbb{C}^{n} \times U_{i}
$$

with the trivial vector bundle such that:

(1) $\psi_{i}$ takes the partial connection $D(V)$ to the partial connection on the trivial vector bundle induced by the natural flat connection on it, and

(2) for any pair $i, j \in L$, the map

$$
\psi_{j} \circ \psi_{i}^{-1}: U_{i} \cap U_{j} \longrightarrow \mathrm{GL}(n, \mathbb{C})
$$

is holomorphic.

A map to $\operatorname{GL}(n, \mathbb{C})$ is called holomorphic if each entry of $n \times n$-matrices defines a holomorphic function. We recall that a locally defined complex valued function $f$ on $M$ is called holomorphic if and only if it is constant along the leaves and the differential $d f$ intertwines the transversely holomorphic structure $J$ and the natural complex structure of $\mathbb{C}$. If $f$ is constant along the leaves, then $d f$ defines a homomorphism from the normal bundle $N$ to $\mathbb{C}$. The condition that $d f$ intertwines the complex structures means that the homomorphism $d f$ is $\mathbb{C}$-linear for the complex structure on $N$ defined by $J$.

The sheaf on $M$ defined by holomorphic functions coincides with the sheaf $\mathcal{C}(0)$ defined earlier. However, we will use the more suggestive notation $\mathcal{O}_{M}$ for the sheaf of holomorphic functions.

\section{Transversal Jet Bundles}

Given a projective structure on a Riemann surface there are some naturally associated differential operators. We will extend the construction of differential operators to the context of transversely projective structures. For that we need to construct, using the foliation, certain subbundles of the jet bundles which we will call transversal jet bundles.

We will begin by recalling the standard properties of a jet bundle. Let $W$ be a $C^{\infty}$ vector bundle over a $C^{\infty}$ manifold $X$. For any integer $k \geq 0$, the $k$ th order jet bundle $J_{r}^{k}(W)$ is a $C^{\infty}$ vector bundle over $X$ whose fiber over any point $x \in X$ is the space of all sections of $W$ over the $k$ th order infinitesimal neighborhood of $x$. Restriction of such sections to the $(k-1)$ th order infinitesimal neighborhood of $x$ defines an exact sequence

$$
0 \longrightarrow S^{k}\left(T^{*} X\right) \otimes W \longrightarrow J_{r}^{k}(W) \longrightarrow J_{r}^{k-1}(W) \longrightarrow 0
$$

of vector bundles, where $S^{k}\left(T^{*} X\right)$ is the $k$ th symmetric power, using the fact that the fiber $\left(S^{k}\left(T^{*} X\right) \otimes W\right)_{x}$ can be identified with the space of (local) sections of $W$ vanishing at $x$ of order $k$ quotiented by sections whose order of vanishing is at least $k+1$.

The subscript $r$ in $J_{r}^{k}$ stands for $C^{\infty}$ (real) jet bundle. Since our main emphasis is on holomorphic jet bundles, the notation $J^{k}$ will be reserved for holomorphic jet bundles.

If $X$ is a complex manifold and $W$ a holomorphic vector bundle, then the subbundle

$$
J^{k}(W) \subset J_{r}^{k}(W)
$$

defined by the holomorphic (local) sections of $W$ has a natural holomorphic structure. 
Let $J$ be a transversely holomorphic structure on the foliation $\mathcal{F}$ on $M$ and $V$ a $C^{\infty}$ complex vector bundle over $M$ equipped with a flat partial connection $D(V)$. Assume that $V$ is equipped with the transversely holomorphic structure. For any point $x \in M$, consider the space of holomorphic sections of $V$ defined around $x$. Its image in the fiber $J_{r}^{k}(V)_{x}$ defines a subspace.

Definition 3.1. For any integer $k \geq 0$, the transversal jet bundle $J_{\mathcal{F}}^{k}(V)$ is the subbundle of the vector bundle $J_{r}^{k}(V)$ whose fiber over any $x \in M$ is the subspace of $J_{r}^{k}(V)_{x}$ defined by the locally defined holomorphic sections of $V$.

Hence, when $\mathcal{F}=0$ (that is, $\operatorname{dim} M=2$ ) the transversal jet bundle $J_{\mathcal{F}}^{k}(V)$ coincides with the holomorphic jet bundle $J^{k}(V)$ defined earlier.

Note that the vector bundle $J_{\mathcal{F}}^{0}(V)$ is identified with $V$. Using this identification, $J_{\mathcal{F}}^{0}(V)$ has a transversely holomorphic structure. In the following lemma we will see that any transversal jet bundle has a transversely holomorphic structure.

Lemma 3.2. For any $k \geq 0$, the transversal jet bundle $J_{\mathcal{F}}^{k}(V)$ has a natural transversely holomorphic structure.

Proof. From the definition of a transversely holomorphic structure we know that there is covering $\left\{U_{j}, \phi_{j}\right\}_{j \in J}$ such that $\mathcal{F}$ is given by the kernel of the differentials of the maps $\phi_{j}: U_{j} \longrightarrow \mathbb{C}$ and there are holomorphic maps $g_{j, j^{\prime}}$ with $g_{j, j^{\prime}} \circ \phi_{j}=\phi_{j^{\prime}}$.

If $W$ is a $C^{\infty}$ vector bundle on the open set $\phi_{j}\left(U_{j}\right) \subset \mathbb{C}$, then $\phi_{j}^{*} W$ has an obvious natural flat partial connection for the foliation $\left.\mathcal{F}\right|_{U_{j}}$ on $U_{j}$. If $W$ has a holomorphic structure, then $\phi_{j}^{*} W$ gets a transversely holomorphic structure on $U_{j}$ in an obvious way.

Using the flat partial connection $D(V)$ on $V$ it follows that for each $j \in J$, there is a complex vector bundle $V_{j}$ on $\phi_{j}\left(U_{j}\right)$ such that $\phi_{j}^{*} V_{j}$ is identified with $\left.V\right|_{U_{j}}$ and the identification takes the partial connections $\left.D(V)\right|_{U_{j}}$ to the natural partial connection on the pullback $\phi_{j}^{*} V_{j}$.

The transversely holomorphic structure on $\left.V\right|_{U_{j}}$ induces a holomorphic structure on $V_{j}$. This holomorphic structure on $V_{j}$ is determined by the condition that the identification of $\phi_{j}^{*} V_{j}$ with $\left.V\right|_{U_{j}}$ takes the given transversely holomorphic structure on $\left.V\right|_{U_{j}}$ to the transversely holomorphic structure on $\phi_{j}^{*} V_{j}$ defined by the holomorphic structure on $V_{j}$.

Now it is easy to see that the restriction of the vector bundle $J_{\mathcal{F}}^{k}(V)$ to the open subset $U_{j}$ is identified with $\phi_{j}^{*} J^{k}\left(V_{j}\right)$. The above defined holomorphic structure on $V_{j}$ induces a holomorphic structure on $J^{k}\left(V_{j}\right)$. This in turn induces a transversely holomorphic structure on $\left.\phi_{j}^{*} J^{k}\left(V_{j}\right) \cong J_{\mathcal{F}}^{k}(V)\right|_{U_{j}}$ over $U_{j}$.

In order to complete the proof, we need to show that the restriction of the transversely holomorphic structure on $\left.J_{\mathcal{F}}^{k}(V)\right|_{U_{j}}$ to $U_{j} \cap U_{j^{\prime}}$ coincides with the restriction of the transversely holomorphic structure on $\left.J_{\mathcal{F}}^{k}(V)\right|_{U_{j}^{\prime}}$. To prove this, first observe that since the holomorphic structure on $V_{j}$ in induced by a transversely holomorphic structure on $V$, for every $j^{\prime} \in J$ there is a holomorphic isomorphism

$$
\tau_{j, j^{\prime}}:\left.\left.V_{j}\right|_{\phi_{j}\left(U_{j} \cap U_{j^{\prime}}\right)} \longrightarrow V_{j^{\prime}}\right|_{\phi_{j^{\prime}}\left(U_{j} \cap U_{j^{\prime}}\right)}
$$


over the obvious map $\sigma_{j, j^{\prime}}: \phi_{j}\left(U_{j} \cap U_{j^{\prime}}\right) \longrightarrow \phi_{j^{\prime}}\left(U_{j} \cap U_{j^{\prime}}\right)$ such that the diagram

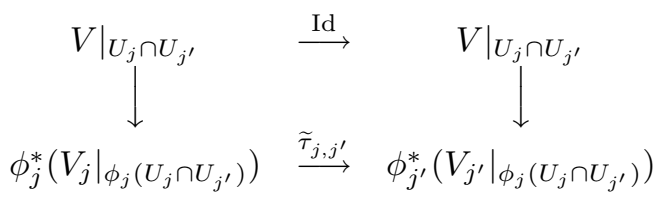

commutes, where $\widetilde{\tau}_{j, j^{\prime}}$ is induced by $\tau_{j, j^{\prime}}$ and the vertical isomorphisms are the natural identifications for $\phi_{j}$ and $\phi_{j^{\prime}}$ respectively. The map $\sigma_{j, j^{\prime}}$, by definition, satisfies the condition $\sigma_{j, j^{\prime}} \circ \phi_{j}=\phi_{j^{\prime}}$ over $U_{i} \cap U_{j}$. Therefore, $\tau_{j, j^{\prime}}$ induces an isomorphism $\widetilde{\tau}_{j, j^{\prime}}$ as above.

The isomorphism $\tau_{j, j^{\prime}}$ induces a holomorphic isomorphism between $\left.J^{k}\left(V_{j}\right)\right|_{\phi_{j}\left(U_{j} \cap U_{j^{\prime}}\right)}$ and $\left.J^{k}\left(V_{j^{\prime}}\right)\right|_{\phi_{j^{\prime}}\left(U_{j} \cap U_{j^{\prime}}\right)}$ over the map $\sigma_{j, j^{\prime}}$. The commutativity of the above diagram implies that this holomorphic isomorphism transports to an identification between the restrictions of the transversely holomorphic structures on $\left.J_{\mathcal{F}}^{k}(V)\right|_{U_{j}}$ and $\left.J_{\mathcal{F}}^{k}(V)\right|_{U_{j}^{\prime}}$ to the intersection $U_{j} \cap U_{j^{\prime}}$. This completes the proof of the lemma.

Let $W_{1}$ and $W_{2}$ be two holomorphic vector bundles on a complex manifold $X$. The sheaf of differential operators of order $k$ from $W_{1}$ to $W_{2}$ is defined to be the sheaf of sections of the vector bundle $\operatorname{Hom}\left(J^{k}\left(W_{1}\right), W_{2}\right)$. In other words, the sheaf of differential operators Diff $X_{X}^{k}\left(W_{1}, W_{2}\right)$ coincides with the sheaf of $\mathcal{O}_{X}$-linear homomorphisms from the sheaf defined by $J^{k}\left(W_{1}\right)$ to the sheaf defined by $W_{2}$. (See 2] for the details.)

Let $V_{1}$ and $V_{2}$ be transversely holomorphic vector bundles over the transversely holomorphically foliated manifold $M$. The sheaf of transversal differential operators of order $k$ from $V_{1}$ to $V_{2}$ is defined to be the sheaf of $\mathcal{O}_{M}$-linear homomorphisms from the sheaf of holomorphic section of $J_{\mathcal{F}}^{k}\left(V_{1}\right)$ to the sheaf of holomorphic sections of $V_{2}$. We recall that $\mathcal{O}_{M}$ is the sheaf defined by holomorphic functions; holomorphic sections of a holomorphic vector bundle were defined in the previous section.

The sheaf of transversal differential operators of order $k$ from $V_{1}$ to $V_{2}$ will be denoted by $\operatorname{Difff}_{\mathcal{F}}^{k}\left(V_{1}, V_{2}\right)$.

In the next section we will see that for each transversely projective foliation there are some associated transversal differential operators.

\section{DifFERENTIAL OPERATORS AND TRANSVERSELy PROJECTIVE FOLIATION}

Consider the jet bundle $J^{2 k}\left(T^{\otimes k}\right)$ over $\mathbb{C P}^{1}:=\mathbb{C} \cup\{\infty\}$, where $T$ denotes the holomorphic tangent bundle. We will show that $J^{2 k}\left(T^{\otimes k}\right)$ has a canonical trivialization. The restriction of any global section of $T^{\otimes k}$ to the $2 k$ th order infinitesimal neighborhoods of points in $\mathbb{C P}^{1}$ define a homomorphism

$$
f_{k}: H^{0}\left(\mathbb{C P}^{1}, T^{\otimes k}\right) \otimes_{\mathbb{C}} \mathcal{O}_{\mathbb{C P}^{1}} \longrightarrow J^{2 k}\left(T^{\otimes k}\right)
$$

from the trivial vector bundle with fiber $H^{0}\left(\mathbb{C P}^{1}, T^{\otimes k}\right)$. This homomorphism $f_{k}$ is an isomorphism. Indeed, both the vector bundles are of rank $2 k+1$ and the homomorphism $f_{k}$ is fiberwise injective.

Since $f_{k}$ in (4.1) is an isomorphism, we have a splitting of the natural exact sequence

$$
0 \longrightarrow T^{\otimes(-k-1)} \longrightarrow J^{2 k+1}\left(T^{\otimes k}\right) \longrightarrow J^{2 k}\left(T^{\otimes k}\right) \longrightarrow 0
$$


for jet bundles given by the homomorphism

$$
J^{2 k}\left(T^{\otimes k}\right) \cong H^{0}\left(\mathbb{C P}^{1}, T^{\otimes k}\right) \otimes_{\mathbb{C}} \mathcal{O}_{\mathbb{C P}^{1}} \longrightarrow J^{2 k+1}\left(T^{\otimes k}\right)
$$

defined by restricting global sections of $T^{\otimes k}$ to the $(2 k+1)$ th order infinitesimal neighborhoods of points. Now the homomorphism

$$
J^{2 k+1}\left(T^{\otimes k}\right) \longrightarrow T^{\otimes(-k-1)}
$$

defined by the splitting of (4.2) gives a differential operator

$$
\mathcal{D}(2 k+1) \in H^{0}\left(\mathbb{C P}^{1}, \operatorname{Diff}_{\mathbb{C P}^{1}}^{2 k+1}\left(T^{\otimes k}, T^{\otimes(-k-1)}\right)\right)
$$

on $\mathbb{C P}^{1}$ of order $2 k+1$. (See [2, (3.6)] for the details of the construction of the operator $\mathcal{D}(2 k+1)$.) The operator $\mathcal{D}(0)$ coincides with the Dolbeault operator $\partial: \mathcal{O}_{\mathbb{C P}^{1}} \longrightarrow T^{*}$.

The group of automorphisms of $\mathbb{C P}^{1}$ coincides with the Möbius group PSL $(2, \mathbb{C})$. Any automorphism of $\mathbb{C P}^{1}$ lifts in an obvious way to an automorphism of any tensor power of $T$. The operator $\mathcal{D}(2 k+1)$ is left invariant by the action of the Möbius group. This follows from the construction of $\mathcal{D}(2 k+1)$ (see [2, Theorem 3.7]).

Let $\mathcal{F}$ be a foliation on $M$ equipped with a transversely projective structure. Therefore, we have a covering $\left\{U_{j}, \phi_{j}\right\}_{j \in J}$ of $M$ such that the kernel of $d \phi_{j}$ defines the foliation on $U_{j}$ and the submersions $\phi_{j}: U_{j} \longrightarrow \mathbb{C}$ differ by Möbius transformations (see Section 21). As we noted in Section 2, the normal bundle $N$ is identified with $\phi_{j}^{*} T \mathbb{C}$ and the identification takes the almost complex structure on the holomorphic tangent bundle $T \mathbb{C}$ to the underlying transversely holomorphic structure for the given transversely projective structure.

Using the identification of $N$ with $\phi_{j}^{*} T \mathbb{C}$, the homomorphism (4.3) over $\phi_{j}\left(U_{j}\right) \subset$ $\mathbb{C P}^{1}$ defines a homomorphism

$$
\beta_{j}: J_{\mathcal{F}}^{2 k+1}(N) \longrightarrow N^{\otimes(-k-1)} .
$$

Now recall the construction, given in Lemma 3.2 of the transversely holomorphic structure on the transversal jet bundle $J_{\mathcal{F}}^{2 k+1}(N)$ of the transversely holomorphic line bundle $N$. From this construction it is immediate that the homomorphism $\beta_{j}$ takes (locally defined) holomorphic sections of $J_{\mathcal{F}}^{2 k+1}(N)$ to holomorphic sections of $N^{\otimes(-k-1)}$.

For any $j^{\prime} \in J$, the two homomorphisms $\beta_{j}$ and $\beta_{j^{\prime}}$ coincides over $U_{j} \cap U_{j^{\prime}}$. Indeed, this is an immediate consequence of the combination of the facts that $\phi_{j}$ and $\phi_{j^{\prime}}$ differ by a Möbius transformation and the homomorphism in (4.3) commutes with the action of the Möbius group on $\mathbb{C P}^{1}$.

Consequently, the collection of homomorphisms $\left\{\beta_{j}\right\}_{j \in J}$ patch together compatibly to give a transversal differential operator

$$
\mathcal{D}_{\mathcal{F}}(2 k+1) \in \Gamma\left(M ; \operatorname{Diff}_{\mathcal{F}}^{2 k+1}\left(N^{\otimes k}, N^{\otimes(-k-1)}\right)\right)
$$

of order $2 k+1$ over $M$ for each $k \geq 0$.

The operator $\mathcal{D}_{\mathcal{F}}(0)$ coincides with the Dolbeault operator $\mathcal{O}_{M} \longrightarrow N^{*}$ defined by the transversely holomorphic structure. In particular, it is independent of the transversely projective structure (depends only on the underlying transversely holomorphic structure). However, for $k \geq 1$, the operator $\mathcal{D}_{\mathcal{F}}(2 k+1)$ depends on the transversely projective structure.

In the next section we will describe deformations of a transversely projective structure using the operator $\mathcal{D}_{\mathcal{F}}(3)$. 


\section{DEFORMATIONS OF A TRANSVERSELY PROJECTIVE STRUCTURE}

In this section, $\mathcal{F}$ will denote a $C^{\infty}$ foliation on $M$ equipped with a transversely projective structure.

As in Section 2, let $\mathcal{C}(1)$ denote the sheaf of holomorphic sections of $N$. Consider the transversal differential operator

$$
\mathcal{D}_{\mathcal{F}}(3) \in \Gamma\left(M ; \operatorname{Diff}_{\mathcal{F}}^{2 k+1}\left(N, N^{\otimes(-2)}\right)\right)
$$

that was constructed in $(4.5)$. Let

$$
\mathcal{W}(\mathcal{F}) \subset \mathcal{C}(1)
$$

be the subsheaf defined by the kernel of the operator $\mathcal{D}_{\mathcal{F}}(3)$. Note that $\mathcal{C}(1)$ depends only on the underlying transversely holomorphic structure of $\mathcal{F}$, but the subsheaf $\mathcal{W}(\mathcal{F})$ depends on the transversely projective structure.

From Lemma 3.2 we know that the transversal jet bundle $J_{\mathcal{F}}^{2}(N)$ has a natural flat partial connection. By a flat connection on $J_{\mathcal{F}}^{2}(N)$ extending the partial connection we mean a flat connection whose restriction to any leaf coincides with the partial connection.

Lemma 5.1. The vector bundle $J_{\mathcal{F}}^{2}(N)$ has a natural flat connection that extends the partial connection. The sheaf $\mathcal{W}(\mathcal{F})$ defined in (5.1) is canonically identified with the sheaf of (locally defined) flat sections of $J_{\mathcal{F}}^{2}(N)$.

Proof. The isomorphism defined by $f_{1}$ in 4.1 of $J^{2}(T)$ over $\mathbb{C P}^{1}$ with a trivial vector bundle induces a flat connection on $J^{2}(T)$. This flat connection on $J^{2}(T)$ will be denoted by $\nabla^{T}$. From its construction it is clear that the connection $\nabla^{T}$ is preserved by the action of the Möbius group on $\mathbb{C P}^{1}$.

The sheaf of solutions of a linear differential operator gives a flat vector bundle. Consider the differential operator $\mathcal{D}(3)$ constructed in 4.4). From its construction it is immediate that the flat vector bundle given by its sheaf of solutions coincides with $J^{2}(T)$ equipped with the connection $\nabla^{T}$. Indeed, since the operator $\mathcal{D}(3)$ is constructed using the splitting of (4.2) defined by the identification of $J^{2}(T)$ with the trivial vector bundle with fiber $H^{0}\left(\mathbb{C P}^{1}, T\right)$, the flat vector bundle $\left(J^{2}(T), \nabla^{T}\right)$ coincides with the one defined by the sheaf of solutions of $\mathcal{D}(3)$.

The flat connection $\nabla^{T}$ on $J^{2}(T)$ induces a flat connection on the transversal jet bundle $J_{\mathcal{F}}^{2}(N)$. To see this, take a covering $\left\{U_{j}, \phi_{j}\right\}_{j \in J}$ of $M$ as in the definition of a transversely projective structure. So each $\phi_{j^{\prime}} \circ \phi_{j}^{-1}$ is a Möbius transformation. The restriction of $J_{\mathcal{F}}^{2}(N)$ to $U_{j}$ is identified with the pullback $\phi_{j}^{*} J^{2}(T)$ (recall the proof of Lemma 3.2). Using this identification, the flat connection $\nabla^{T}$ on $J^{2}(T)$ induces a flat connection on $\left.J_{\mathcal{F}}^{2}(N)\right|_{U_{j}}$ over $U_{j}$. Let $g_{j^{\prime}, j}$ denote the element of the Möbius group $\operatorname{PSL}(2, \mathbb{C})$ that coincides with $\phi_{j^{\prime}} \circ \phi_{j}^{-1}$. The action of $g_{j^{\prime}, j}$ on $\mathbb{C P}^{1}$ preserves the flat connection $\nabla^{T}$. In other words, the above defined connections on $\left.J_{\mathcal{F}}^{2}(N)\right|_{U_{j}}$ and $\left.J_{\mathcal{F}}^{2}(N)\right|_{U_{j^{\prime}}}$ coincide over $U_{j} \cap U_{j^{\prime}}$. Therefore, we have a flat connection on $J_{\mathcal{F}}^{2}(N)$ over $M$.

This flat connection on $J_{\mathcal{F}}^{2}(N)$ will be denoted by $\nabla^{\mathcal{F}}$. Since the canonical identification of $\left.J_{\mathcal{F}}^{2}(N)\right|_{U_{j}}$ with $\phi_{j}^{*} J^{2}(T)$ takes the restriction $\left.\nabla^{\mathcal{F}}\right|_{U_{j}}$ to the pullback of a connection on $J^{2}(T)$ over $\phi_{j}\left(U_{j}\right)$, it follows immediately that $\nabla^{\mathcal{F}}$ is an extension of the natural partial connection on $J_{\mathcal{F}}^{2}(N)$.

We already observed that the flat vector bundle given by the solutions of $\mathcal{D}(3)$ coincides with the flat bundle $\left(J^{2}(T), \nabla^{T}\right)$. From the constructions of the operator 
$\mathcal{D}_{\mathcal{F}}(3)$ and the connection $\nabla^{\mathcal{F}}$ it is immediate that over any $U_{j}$, the restriction of the solution sheaf $\mathcal{W}(\mathcal{F})$ (defined in (5.1)) is identified with the sheaf of flat sections of the connection $\left.\nabla^{\mathcal{F}}\right|_{U_{j}}$. These identifications patch compatibly (as any $\phi_{j^{\prime}} \circ \phi_{j}^{-1}$ is a Möbius transformation and $\mathcal{D}(3)$ is left invariant by the action of the Möbius group on $\left.\mathbb{C P}^{1}\right)$, and hence we get an isomorphism of $\mathcal{W}(\mathcal{F})$ with the sheaf of flat sections for the flat connection $\nabla^{\mathcal{F}}$. This completes the proof of the lemma.

The following proposition describes the homomorphism $\delta$ mentioned in the introduction.

Proposition 5.2. There is a natural homomorphism

$$
\delta: H^{i}(M, \mathcal{W}(\mathcal{F})) \longrightarrow H^{1}(M, \mathcal{C}(1))
$$

for every $i \geq 0$, where $\mathcal{C}(1)$ is the sheaf of holomorphic sections of $N$.

Proof. In 5.1 we saw that $\mathcal{W}(\mathcal{F})$ is a subsheaf of $\mathcal{C}(1)$. This inclusion map of sheaves induces a homomorphism $\delta$ as in the statement of the proposition. We will give an alternative description of this homomorphism $\delta$ to show that it is actually a part of a long exact sequence.

The operator $\mathcal{D}_{\mathcal{F}}(3)$ is surjective in the sense that given a holomorphic section $s$ of $N^{\otimes(-2)}$ around a point $x \in M$, there is a sufficiently small neighborhood $U$ of $x$ and a holomorphic section $w$ of $N$ over $U$ such that $\mathcal{D}_{\mathcal{F}}(3)(w)=s$ over $U$. Indeed, this surjectivity property of $\mathcal{D}_{\mathcal{F}}(3)$ follows from the same property of the operator $\mathcal{D}(3)$ over $\mathbb{C P}^{1}$ constructed in (4.4).

Therefore, the sheaf $\mathcal{W}(\mathcal{F})$ is quasi-isomorphic to the two term complex

$$
C_{0}:=\mathcal{C}(1) \stackrel{\mathcal{D}_{\mathcal{F}}(3)}{\longrightarrow} C_{1}:=\mathcal{C}(-2)
$$

which will be denoted by $C$. for short. In other words, $H^{i}(M, \mathcal{W}(\mathcal{F}))$ is identified with the hypercohomology $\mathbb{H}^{i}(C$. $)$.

Now, the complex $C$. fits in the following short exact sequence of complexes

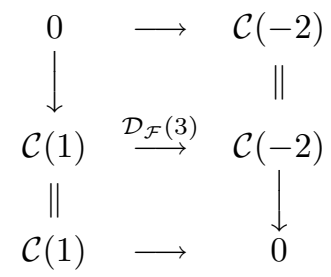

over $M$. Note that both the top and the bottom complexes have only one nonzero term. The $i$ th hypercohomology of the top (respectively, bottom) complex coincides with $H^{i-1}(M, \mathcal{C}(-2))$ (respectively, $H^{i}(M, \mathcal{C}(1))$ ).

Consider the long exact sequence of hypercohomologies

$$
\begin{aligned}
\cdots H^{i-1}(M, \mathcal{C}(-2)) & \longrightarrow \mathbb{H}^{i}(C .) \longrightarrow H^{i}(M, \mathcal{C}(1)) \\
& \longrightarrow H^{i}(M, \mathcal{C}(-2)) \longrightarrow \mathbb{H}^{i+1}(C .) \cdots
\end{aligned}
$$

for the exact sequence (5.3). The above homomorphism $\mathbb{H}^{i}(C$. $) \longrightarrow H^{i}(M, \mathcal{C}(1))$ coincides with the earlier defined homomorphism $\delta$ using the identification of $\mathbb{H}^{i}(C$.) with $H^{i}(M, \mathcal{W}(\mathcal{F}))$.

Let $\mathcal{F}^{\prime}$ denote the transversely holomorphic foliation underlying the transversely projective foliation $\mathcal{F}$. There is a natural homomorphism from the space of all infinitesimal deformations of the transversely holomorphic foliation $\mathcal{F}^{\prime}$ to $H^{1}(M, \mathcal{C}(1))$ 
(see [6] Section 4]). Under the assumption that $M$ is compact, in 6, Section 4, p. $178]$ it is shown that this homomorphism is injective and its image is identified. The infinitesimal deformations of the almost complex structure on the normal bundle $N$ are parametrized by flat sections of $\operatorname{Hom}(\bar{N}, N)$ for the Bott partial connection. The Dolbeault resolution of $\mathcal{C}(1)$ gives a homomorphism from the space of such sections to $H^{1}(M, \mathcal{C}(1))$.

Let $\mathcal{I}^{\prime}$ denote the space of all infinitesimal deformations of the transversely holomorphic foliation $\mathcal{F}^{\prime}$, and let

$$
\zeta^{\prime}: \mathcal{I}^{\prime} \longrightarrow H^{1}(M, \mathcal{C}(1))
$$

be the above mentioned homomorphism.

The space of all infinitesimal deformations of the transversely projective foliation $\mathcal{F}$ will be denoted by $\mathcal{I}$. If we associate to any transversely projective foliation the underlying transversely holomorphic foliation, then this induces a homomorphism of the corresponding infinitesimal deformations. In other words, we have a homomorphism

$$
f: \mathcal{I} \longrightarrow \mathcal{I}^{\prime}
$$

induced by the forgetful map that sends any transversely projective foliation to its underlying transversely holomorphic foliation.

Theorem 5.3. There is a natural homomorphism $\zeta: \mathcal{I} \longrightarrow H^{1}(M, \mathcal{W}(\mathcal{F}))$. Furthermore, the composition $\delta \circ \zeta$ coincides with $\zeta^{\prime} \circ f$, where $\delta, \zeta^{\prime}$ and $f$ are constructed in Proposition 5.2, (5.4) and (5.5) respectively.

Proof. We will first show that the fibers of the flat vector bundle $J_{\mathcal{F}}^{2}(N)$ have a Lie algebra structure compatible with the flat connection.

In Lemma 5.1, the flat connection on $J_{\mathcal{F}}^{2}(N)$ was constructed using the flat connection on $J^{2}(T)$ over $\mathbb{C P}^{1}$. The flat connection on $J^{2}(T)$ is induced, using the isomorphism $f_{1}$ defined in (4.1), by the flat connection on the trivial vector bundle $H^{0}\left(\mathbb{C P}^{1}, T\right) \otimes_{\mathbb{C}} \mathcal{O}_{\mathbb{C P}^{1}}$. The Lie bracket operation on the vector fields makes the space $H^{0}\left(\mathbb{C P}^{1}, T\right)$ of global vector fields into a Lie algebra. It is easy to see that this Lie algebra is isomorphic to $\mathrm{sl}(2, \mathbb{C})$. Indeed, the action of the Möbius group $\operatorname{PSL}(2, \mathbb{C})$ on $\mathbb{C P}^{1}$ gives a Lie algebra homomorphism from its Lie algebra $\mathrm{sl}(2, \mathbb{C})$ to $H^{0}\left(\mathbb{C P}^{1}, T\right)$. This homomorphism is easily seen to be an isomorphism. Therefore, the fibers of $J^{2}(T)$ are equipped with a Lie algebra structure compatible with the flat connection (a multiplication of flat sections remains flat).

The lift of the action of the Möbius group $\operatorname{PSL}(2, \mathbb{C})$ on $\mathbb{C P}^{1}$ to $J^{2}(T)$ preserves the Lie algebra structure of the fibers. Therefore, the fibers of the transversal jet bundle $J_{\mathcal{F}}^{2}(N)$ have a Lie algebra structure compatible with the flat connection. Indeed, this is an immediate consequence of the fact that for any map $\phi$ of an open subset $U$ of $M$ to $\mathbb{C}$ compatible with the given transversely projective structure, the restriction of $J_{\mathcal{F}}^{2}(N)$ to $U$ is identified with $\phi^{*} J^{2}(T)$ (see the proof of Lemma 5.1). Note that any fiber of $J_{\mathcal{F}}^{2}(N)$ is isomorphic, as a Lie algebra, to $\operatorname{sl}(2, \mathbb{C})$.

Let $\mathcal{I}^{\prime \prime}$ denote the space of all infinitesimal deformations of the flat Lie algebra bundle $J_{\mathcal{F}}^{2}(N)$. The map that associates to any transversely projective structure $\mathcal{F}_{1}$ the corresponding flat Lie algebra bundle $J_{\mathcal{F}_{1}}^{2}(N)$ defines a homomorphism

$$
\gamma: \mathcal{I} \longrightarrow \mathcal{I}^{\prime \prime} \text {. }
$$

In other words, $\gamma$ is the differential (infinitesimal version) of the above map that sends $\mathcal{F}_{1}$ to the flat Lie algebra bundle $J_{\mathcal{F}_{1}}^{2}(N)$. 
It is a general fact that there is a natural homomorphism from the space of infinitesimal deformations of a flat Lie algebra bundle, with fibers isomorphic to a semisimple Lie algebra, to the first cohomology of the corresponding local system. In other words, we have a homomorphism

$$
\zeta^{\prime \prime}: \mathcal{I}^{\prime \prime} \longrightarrow H^{1}(M, \mathcal{W}(\mathcal{F})) \text {. }
$$

The homomorphism $\zeta$ in the statement of theorem is the composition $\zeta^{\prime \prime} \circ \gamma$, where $\gamma$ and $\zeta^{\prime \prime}$ are constructed in (5.6) and (5.7) respectively.

We will recall the construction of the key map $\zeta^{\prime \prime}$. Fix a complex semisimple Lie algebra $\mathfrak{g}$. A flat Lie algebra bundle on a manifold $X$ with fibers isomorphic to $\mathfrak{g}$ is obtained from a 1-cocycle on $X$ with values in the group $\operatorname{Aut}(\mathfrak{g})$, the Lie algebra preserving linear automorphisms of $\mathfrak{g}$. Let $\left\{U_{j}\right\}_{j \in J}$ be a covering of $X$ by open subsets such that all $U_{j}$ and the intersections $U_{j} \cap U_{j^{\prime}}$ are contractible. Suppose that for each ordered pair $j, j^{\prime} \in J$ we have an automorphism $G_{j, j^{\prime}} \in \operatorname{Aut}(\mathfrak{g})$ satisfying the following conditions:

(1) $G_{j, j}=\operatorname{Id}_{\mathfrak{g}}$

(2) $G_{j, j^{\prime}}=G_{j^{\prime}, j}^{-1}$,

(3) $G_{j, j^{\prime}} G_{j^{\prime}, j^{\prime \prime}} G_{j^{\prime \prime}, j}=\operatorname{Id}_{\mathfrak{g}}$

(equivalently, the collection $\left\{G_{j, j^{\prime}}\right\}_{j, j^{\prime} \in J}$ form a 1-cocycle). Then we obtain a flat Lie algebra bundle as follows. Consider the collection $\left\{U_{j} \times \mathfrak{g}\right\}_{j \in J}$ of trivial $\mathfrak{g}$ bundles. On each $U_{j} \cap U_{j^{\prime}}$, attach $U_{j} \times \mathfrak{g}$ and $U_{j^{\prime}} \times \mathfrak{g}$ using the automorphism $G_{j, j^{\prime}}$ of $\mathfrak{g}$. This gives a flat Lie algebra bundle over $X$. Each flat Lie algebra bundle, with fibers isomorphic to $\mathfrak{g}$, arises this way.

This way, the space of flat Lie algebra bundles over $X$, with fibers isomorphic to $\mathfrak{g}$, gets identified with the first cohomology $H^{1}(X, \operatorname{Aut}(\mathfrak{g}))$.

Now note that the Lie algebra of the Lie group $\operatorname{Aut}(\mathfrak{g})$ is canonically identified with $\mathfrak{g}$ with the identification defined by the adjoint action of $\mathfrak{g}$ on itself. Since $\mathfrak{g}$ is a complex semisimple Lie algebra, if $G$ is a connected Lie group with Lie algebra $\mathfrak{g}$, then the group of outer automorphisms of $\mathfrak{g}$ is a finite group. The group of outer automorphisms is the quotient $\operatorname{Aut}(\mathfrak{g}) / G$, where $G$ acts on $\mathfrak{g}$ by conjugation. Therefore, the Lie algebra of $\operatorname{Aut}(\mathfrak{g})$ is identified with the Lie algebra of $G$.

It is easy to see that the tangent space $T_{\rho} H^{1}(X, \operatorname{Aut}(\mathfrak{g}))$ at any $\rho \in$ $H^{1}(X, \operatorname{Aut}(\mathfrak{g}))$ has a natural homomorphism to $H^{1}\left(X, V_{\rho}\right)$, where $V_{\rho}$ is the local system defined by the flat Lie algebra bundle corresponding to $\rho$.

This describes the homomorphism $\zeta^{\prime \prime}$. Now set $\zeta:=\zeta^{\prime \prime} \circ \gamma$. We want to show that $\zeta$ satisfies the identity $\delta \circ \zeta=\zeta^{\prime} \circ f$.

In the proof of Proposition 5.2 , it was observed that $H^{1}(M, \mathcal{W}(\mathcal{F}))$ is identified with the hypercohomology $\mathbb{H}(C$. $)$. We recall that the homomorphism $\delta$ is obtained from the long exact sequence of hypercohomologies for the exact sequence (5.3). From this it follows that $\delta \circ \zeta$ coincides with the cohomology class representing the deformation of the underlying transversely holomorphic foliation. This completes the proof of the theorem.

\section{REFERENCES}

[1] I. Biswas, Transversely projective structures on a transversely holomorphic foliation, Conform. Geom. Dyn. 5 (2001), 74-80.

[2] I. Biswas, Differential operators on complex manifolds with a flat projective structure, $J$. Math. Pures Appl. 78 (1999), 1-26. MR 99m:14034 
[3] C. Camacho and B. A. Scárdua, Holomorphic foliations and Kupka singular sets, Comm. Anal. Geom. 7 (1999), 623-640. MR 2000f:32043

[4] T. Duchamp and M. Kalka, Deformation theory for holomorphic foliations, J. Differential Geom. 14 (1979), 317-337. MR 82b:57019

[5] J. Girbau, A. Haefliger and D. Sundararaman, On deformations of transversely holomorphic foliations, J. Reine Angew. Math. 345 (1983), 122-147. MR 84j:32026

[6] X. Gómez-Mont, Transversal holomorphic structures, J. Differential Geom. 15 (1980), 161185. MR 82j:53065

[7] X. Gómez-Mont, The transverse dynamics of a holomorphic flow, Ann. of Math. 127 (1988), 49-92. MR 89d:32049

[8] R. C. Gunning, Affine and projective structures on Riemann surfaces, Riemann surfaces and related topics: Proceedings of the 1978 Stony Brook Conference (State Univ. New York, Stony Brook, N.Y., 1978), pp. 225-244, Ann. of Math. Stud., 97, Princeton Univ. Press, Princeton, N.J., 1981. MR 83g:30054

[9] A. Haefliger, Homotopy and integrability. Manifolds-Amsterdam 1970 (Proc. Nuffic Summer School) pp. 133-163, Lecture Notes in Mathematics, Vol. 197 Springer, Berlin, 1971. MR 44:2251

[10] B. A. Scárdua, Transversely affine and transversely projective holomorphic foliations, Ann. Sci. École Norm. Sup. 30 (1997), 169-204. MR 97k:32049

School of Mathematics, Tata Institute of Fundamental Research, Homi Bhabha RoAD, BOMBay 400005, INDIA

E-mail address: indranil@math.tifr.res.in 